\title{
Single-breath-hold whole-heart coronary MRA in healthy volunteers at 3.0-T MRI
}

\author{
Yuji Iyama ${ }^{*}$, Takeshi Nakaura', Masafumi Kidoh², Tetsuya Kawahara', Naritsugu Sakaino ${ }^{3}$, Kazunori Harada ${ }^{4}$, \\ Tomoyuki Okuaki ${ }^{5}$ and Yasuyuki Yamashita ${ }^{6}$
}

\begin{abstract}
Background: The purpose of this study was to investigate the feasibility of single-breath-hold whole-heart MRA with a 3-T system. Ten healthy male volunteers underwent single-breath-hold whole-heart coronary MRA at 3 T. We assessed acquisition time, scores of image quality of coronary artery (RCA: proximal, middle and distal, LAD: main, proximal, middle and distal, LCX: proximal and distal) and the visualized vessel length of RCA, LAD and LCX.
\end{abstract}

Findings: Mean acquisition time was $37.7 \pm 5.2 \mathrm{sec}$. Coronary branch was successfully depicted in 67/80 branches (84\%) in the 10 healthy volunteers with diagnostic image quality. And, the average visible RCA, LAD and LCX vessel length were $83.4 \pm 22 \mathrm{~mm}$ and $59.6 \pm 24 \mathrm{~mm}$.

Conclusions: 3-T MRI with single-breath-hold 3D whole-heart coronary MRA can yield adequate image quality. Further study is needed to evaluate the clinical benefit of this technique.

Keywords: Single-breath-hold whole-heart MRA; Imaging; 3-T MRI

\section{Introduction}

Whole-heart coronary MR angiography (MRA) is generally carried out during free breathing with a respiratory gating method using navigator echo techniques, which track the motion of the right hemi-diaphragmatic dome. The advantage of free breathing technic is unnecessary to stop breathing. However, the major drawback of this free breathing technique is the relatively long acquisition time, ranging from 10 to 20 minutes (Sakuma et al. 2005). The technic of single-breath hold technic could shorten the total scan time because it can reduce influence of respiratory motion. There were some reports about whole-heart coronary MRA with 1.5 T MRI during single breath hold (Makowski et al. 2012; Okada et al. 2011; Nassenstein et al. 2008). In general, increased SNR by $3-T$ MRI enables us to increase image quality and exam speed compared to 1.5-T MRI; however, high-field systems still pose challenges in terms of their specific absorption rate (SAR) and radiofrequency (RF) excitation uniformity, especially in cardiac MRI (Nezafat et al. 2006).

\footnotetext{
* Correspondence: iyamayuuji@yahoo.co.jp

1Diagnostic Radiology, Amakusa Medical Center, Kameba 854-1, Amakusa, Kumamoto 863-0046, Japan

Full list of author information is available at the end of the article
}

Recently introduced 3-T systems encompass two major innovative technologies for coronary MRA. The Direct Digital RF receiver technology digitizes the MR signal at the patient, and the fiber-optic connection from the coil to the image reconstructor enables lossless broadband data transmission. Previous reports suggested that this technique improved the dynamic range of the RF receiver and resulted in an improved signal-to-noise ratio (SNR) (Ruipeng et al. 2009). Dual-source radiofrequency transmission with patient-adaptive local radiofrequency shimming enables uniform RF shimming for cardiac MRI (Mueller et al. 2012). This technique reduces dielectric shading, improves B 1 homogeneity, and increases image contrast by T2 preparation prepulse (T2prep) with high-power refocusing pulses. These new technologies in 3-T MRI might increase the SNR at cardiac MRI, and enable us to shorten the acquisition time of whole-heart coronary MRA with adequate image quality. However, to our knowledge, there is no published protocol for single-breathhold whole-heart MRA with recent 3-T systems.

The purpose of this study was to investigate the feasibility of single-breath-hold whole-heart MRA with a 3-T system on healthy volunteers. (c) 2014 lyama et al.; licensee Springer. This is an Open Access article distributed under the terms of the Creative Commons Attribution License (http://creativecommons.org/licenses/by/4.0), which permits unrestricted use, distribution, and reproduction in any medium, provided the original work is properly credited. 


\section{Methods \\ Subjects}

This prospective study received Amakusa medical center institutional review board approval, and prior informed consent to participate was obtained from 10 healthy male volunteers. All volunteers were imaged consecutively between August 2012 and September 2012. Ages ranged from 24 to 53 (mean $35.6 \pm 11.0$ ) years old, and heart rate ranged from 45 to 75 (mean $59.8 \pm 9.6$ ) beats per minute. Table 1 shows the characteristics of the volunteers.

\section{MR angiography acquisition}

All ten subjects were imaged by 3-T MRI (Ingenia, Philips Medical Systems) using a 16-element phased-array Direct Digital RF receiver coil and vector electrocardiographic (VCG) gating (Fischer et al. 1999). A multi-slice gradient echo $\left(\mathrm{TR}=2.6 \mathrm{~ms} ; \mathrm{TE}=1.27 \mathrm{~ms} ; \alpha=20^{\circ}\right.$ ) scout scan was acquired in 3 orthogonal orientations for localization of the volume for whole-heart imaging. After the 3D scout scan, an axial ECG-triggered, segmented steady-state free precession (SSFP) cine image series $(\mathrm{TR}=2.6 \mathrm{~ms}$, $\mathrm{TE}=1.28 \mathrm{~ms}, \alpha=45^{\circ}$, and temporal resolution of $10 \mathrm{~ms})$ at the level of the proximal-to-mid right coronary artery (RCA) was also obtained during a single breath hold. This was carried out for visual determination of the most quiescent period in the cardiac cycle, which was subsequently used to set the trigger delay and the shot duration. In addition, an ECG-triggered segmented 3D SSFP sequence using the proposed undersampling scheme was implemented and in-vivo measurements were performed in expiration.

Subsequently, 3D whole-heart turbo field echo (TFE) coronary MRA was acquired using this visually identified trigger delay and shot duration. We visually measured the rest period of the RCA during diastole phase, and defined the shot duration as long as possible. No intravenous contrast agents were used; and, a T2prep ( $\mathrm{TE}=70 \mathrm{~ms})$ was used to increase the contrast of natural T2 differences between blood and myocardium. The T2prep technic could yield four times more refocusing pulses than that of without T2 prep technic for turbo gradient echo coronary MRA in 3-T MRI. Spectrally selective fat saturation was also utilized for additional endogenous image contrast enhancement between the coronary blood pool and the surrounding fat. The detailed scanning parameters are shown in Table 2.

\section{Image analysis}

To evaluate the image quality, we performed qualitative image analysis of axial images and curved MPR images on a PACS viewer (Synapse, Fuji Film Medicals). Two board-certified radiologists with 8 and 5 years of experience with cardiac MRI independently graded overall image quality according to a segmentation scheme recommended by the American College of Cardiology and the American Heart Association (ACC/AHA) (Scanlon et al. 1999). The RCA was subdivided into three segments (proximal, middle and distal), the LAD into four segments (main, proximal, and middle) and the LCX into two segments (proximal and distal). We defined these segment as follows; (\#1 in AHA: RCA proximal segment, \#2 in AHA: RCA middle segment, \#3 in AHA: RCA distal segment, \#5 in AHA: LAD main segment, \#6 in AHA: LAD proximal segment, \#7 in AHA: LAD middle segment, \#11 in AHA: LCX proximal segment and \#13 in AHA: LCX distal segment). We used a 5-point subjective scale for qualitative image analysis: 4 , excellent (the vessel was well depicted with sharply defined borders); 3 , good (the vessel was adequately visualized, with confidence in the diagnosis, only mildly blurred borders); 2 , fair (coronary vessel was visible, but confidence in the diagnosis was low due to moderately blurred borders); 1 , poor (coronary vessel was barely seen or was obscured by noise); and 0 , not visualized (Sakuma et al. 2005; Wu et al. 2007). The

Table 1 Volunteer's objective data

\begin{tabular}{|c|c|c|c|c|c|c|c|c|c|}
\hline Number & Sex & Age (years) & HR (beats/min) & Total scan time (sec) & Height $(\mathrm{cm})$ & Weight (kg) & LAD & LCX & RCA \\
\hline 1 & Male & 47 & 60 & 45 & 172 & 67 & 98.9 & 53.7 & 131.3 \\
\hline 2 & Male & 53 & 75 & 45 & 171 & 76 & 116.8 & 77.6 & 118 \\
\hline 3 & Male & 46 & 60 & 40 & 168 & 72 & 75.8 & 20.3 & 130.2 \\
\hline 4 & Male & 24 & 75 & 38 & 167 & 63 & 49.1 & 76.9 & 77.8 \\
\hline 5 & Male & 31 & 55 & 34 & 174 & 58 & 75.4 & 37.9 & 140.9 \\
\hline 6 & Male & 28 & 50 & 31 & 175 & 65 & 97.9 & 74.5 & 97.9 \\
\hline 7 & Male & 45 & 55 & 32 & 168 & 76 & 95.2 & 86.1 & 80.4 \\
\hline 8 & Male & 24 & 45 & 40 & 166 & 61 & 115 & 55.2 & 148 \\
\hline 9 & Male & 25 & 60 & 40 & 175 & 63 & 90.6 & 83 & 76.3 \\
\hline 10 & Male & 33 & 63 & 32 & 170 & 63 & 57.8 & 49.4 & 148 \\
\hline average & & $35.6 \pm 11.0$ & $59.8 \pm 9.6$ & $37.7 \pm 5.2$ & $170.6 \pm 3.3$ & $66.4 \pm 6.3$ & $87.3 \pm 22.5$ & $61.5 \pm 21.7$ & $114.9 \pm 29.3$ \\
\hline
\end{tabular}

Note: Data are shown as the mean \pm standard deviation. 


\section{Table 2 Scan parameters}

\begin{tabular}{lc}
\hline FOV & $320 \mathrm{~mm}$ \\
RFOV & $80 \%$ \\
ACQ voxel size & $2.0 \times 2.0 \times 2.0 \mathrm{~mm}$ \\
Reconstructed voxel size & $1.0 \times 1.0 \times 1.0 \mathrm{~mm}$ \\
Slices & 124 \\
Slice thickness & $2 \mathrm{~mm}(1 \mathrm{~mm}$ reconstruction $)$ \\
Scan mode & $3 \mathrm{D}$ \\
Scan technique & FFE \\
Fast imaging mode & TFE \\
Shot mode & Multishot \\
Profile order & Low-high \\
Turbo direction & Radial \\
TR/TE & $3.4 / 1.5 \mathrm{msec}$ \\
Fat suppression & SPIR \\
NSA & 1 \\
Half-scan & Y factor: 0.625, Z factor: 0.9 \\
Flip angle & 12 deg. \\
T2prep & TE: 70 ms, 4 RF pulses \\
SENSE factor & Phase direction 2, Slice direction 1.5 \\
\hline
\end{tabular}

visualized vessel length of RCA, LAD and LCX were also measured. Interobserver disagreements were resolved by consensus.

\section{Results}

Single-breath-hold whole-heart coronary MRA was technically successful in all 10 volunteers. Total scan times varied from $31 \mathrm{sec}$ to $45 \mathrm{sec}$ because we changed the shot duration for each volunteer.

The TFE sequence provided uniform, depictions of coronary arteries, as shown in Table 3 . Table 3 show the results of the qualitative analysis. Coronary branch was successfully depicted in $62 / 80$ branches $(77.5 \%)$ by reader $1,66 / 80$ branches (82.5\%) by reader 2 and $67 / 80$ branches
(83.8\%) by consensus in the 10 healthy volunteers with diagnostic image quality (score: 4 or 3 ). The average visible RCA, LAD and LCX vessel length were $114.9 \pm$ $29.3 \mathrm{~mm}, 83.4 \pm 22 \mathrm{~mm}$ and $59.6 \pm 24 \mathrm{~mm}$. Figure 1 shows an image (original image, curved MPR and PWMIP) of a volunteer, for whom three coronary branches were successfully depicted with diagnostic image quality.

\section{Findings}

The purpose of this study was to investigate the feasibility of single-breath-hold whole-heart MRA with a 3-T system. We assessed acquisition time, scores of image quality of coronary artery of ten volunteers. Mean acquisition time was $37.7 \pm 5.2 \mathrm{sec}$. The average visible RCA, LAD and LCX vessel length were $83.4 \pm 22 \mathrm{~mm}$ and $59.6 \pm 24 \mathrm{~mm}$.

\section{Discussion}

To our knowledge, this is the first report on the clinical feasibility of single-breath-hold 3D whole-heart coronary MRA in 3-T MRI.

Although single-breath-hold 3D whole-heart coronary MRA has been performed at 1.5-T MRI using the SSFP technique, the increased B1 field inhomogeneity and SAR limit the consistency of SSFP in coronary images in 3.0-T MRI (Nezafat et al. 2006; Stuber et al. 2002). There are a few reports about $1.5 \mathrm{~T}$ single-breath-hold coronary MRA (Makowski et al. 2012; Lim et al. 2013). Because, this technic has disadvantage of breathing hold for a long time, therefore, patients with a respiratory disease have difficulty in receiving examination. However, 3 T MRI can shorten scan time, and patients may not need a long breath-hold. Therefore, TFE sequence that has better tolerance to field inhomogeneity than SSFP has been used for coronary MRA at 3.0 T. However, a major drawback of coronary MRA with TFE is that the SNR of the coronary arteries and the blood-myocardial contrast are not as high as those of SSFP sequence (Maintz et al. 2004). And, there were few reports about single-breath-hold 3D whole-heart coronary MRA with our study suggested that a recent

Table 3 Image analysis

\begin{tabular}{lccc}
\hline & Reader 1 & Reader 2 & Consensus \\
\hline Well-depicted coronary branch (score: 4 or 3) & $62 / 80(77.5 \%)$ & $66 / 80(82.5 \%)$ & $67 / 80(83.8 \%)$ \\
RCA proximal segment (\#1) & $3.5 \pm 0.7$ & $3.7 \pm 0.7$ & $3.6 \pm 0.7$ \\
RCA middle segment (\#2) & $3.5 \pm 0.7$ & $3.7 \pm 0.7$ & $3.6 \pm 0.7$ \\
RCA distal segment (\#3) & $2.5 \pm 0.7$ & $3.7 \pm 0.7$ & $3.1 \pm 1.0$ \\
LAD main segment (\#5) & $2.7 \pm 0.5$ & $3.6 \pm 0.7$ & $3.3 \pm 0.7$ \\
LAD proximal segment (\#6) & $2.4 \pm 0.5$ & $3.5 \pm 0.7$ & $3.1 \pm 0.7$ \\
LAD middle segment (\#7) & $2.7 \pm 0.5$ & $3.3 \pm 0.9$ & $3.0 \pm 0.7$ \\
LCX proximal segment (\#11) & $2.8 \pm 0.6$ & $3.2 \pm 0.9$ & $3.2 \pm 0.8$ \\
LCX distal segment (\#13) & $2.7 \pm 0.5$ & $3.3 \pm 0.9$ & $3.1 \pm 0.7$ \\
\hline
\end{tabular}

Note: Data are shown as the mean \pm standard deviation. 


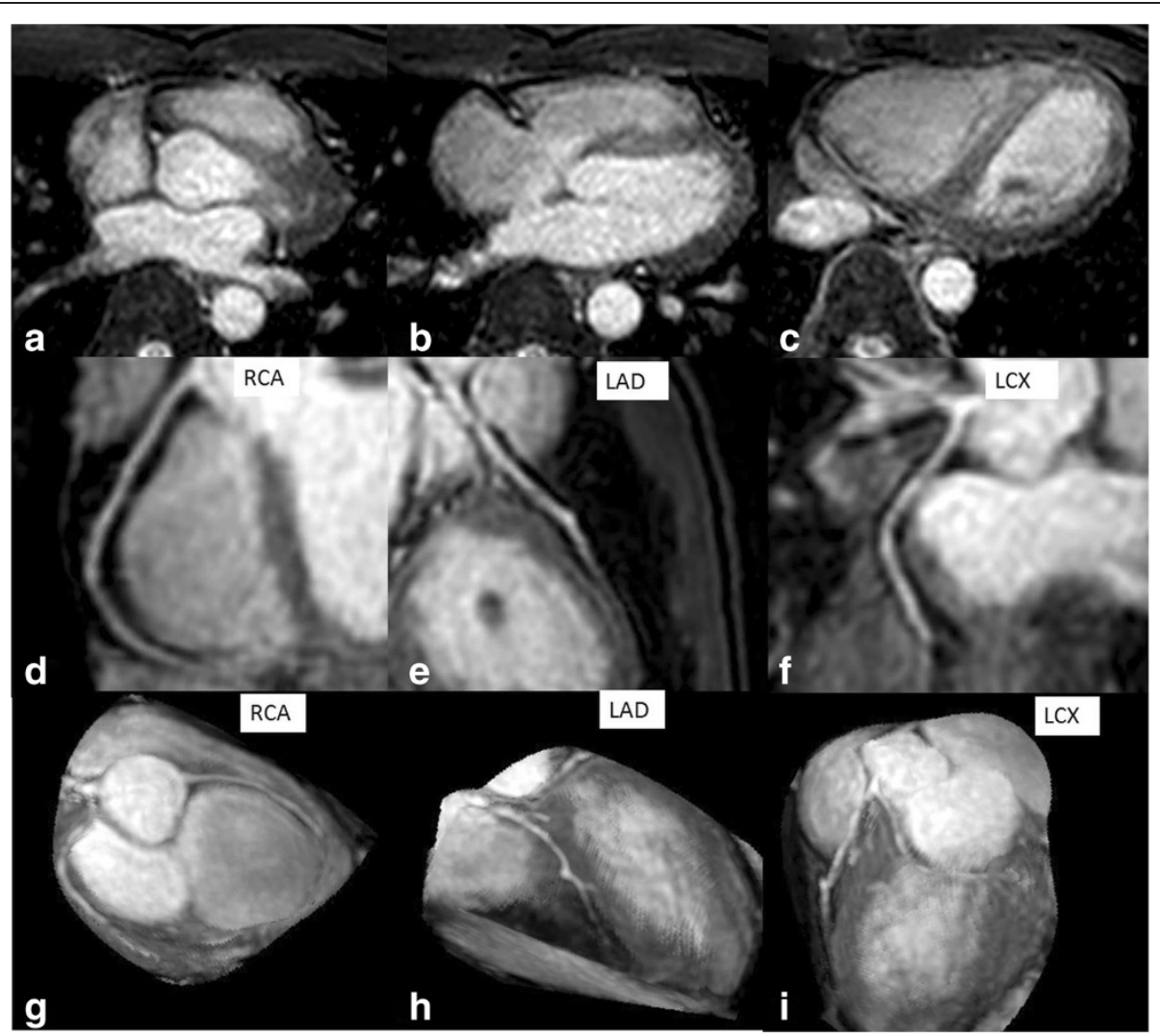

Figure 1 A 24-year-old volunteer was imaged by single-breath-hold whole-heart MRA with a 3-T system. His heart rate was 51 beats per minute, shot duration time $150 \mathrm{~ms}$ and scan time $31 \mathrm{sec}$ (volunteer number 8 in Table 1). We showed his original image (a, b, and c), curved MPR (multi planner reconstruction) image (d, e, and $\mathbf{f}$ ) and PWMIP (partial width maximum intensity projection) image (g, $\mathbf{h}$, and $\mathbf{i})$. All coronary branches were successfully depicted with diagnostic image quality. The qualitative image score is (RCA proximal segment: 4, RCA middle segment: 4, RCA distal segment: 4, LAD main segment: 4, LAD proximal segment: 3, LAD middle segment: 3, LCX proximal segment: 4, LCX distal segment: 4).

3-T MRI might offer adequate SNR in spite of having the short acquisition time of whole-heart coronary MRA. We did not know the reason the result of our study was better than that of previous report about single-breath-hold 3D whole-heart coronary MRA (Nezafat et al. 2006; Stuber et al. 2002). There are many factors to improve image quality ( for example, recent 3-T MRI system, such as increasing the number of channel coils, the Direct Digital RF receiver technology and the dual-source radiofrequency transmission). We think that the increased refocusing pulses (T2prep) mainly overcome the decreased signal with the TFE sequence in free breathing coronary MRI. In general, the image contrast upon using TFE MRI is dependent on the number of prepulses. Botnar et al. state that the combined approach of freebreathing navigator-gated and slice-tracked 3D coronary MRA together with a T2prep and a shorter acquisition window resulted in an improved CNR between coronary blood and myocardium and thereby allowed for better definition of the coronary vessels (Maintz et al. 2004). And, T2 prep technic may be useful in breath-hold coronary MRI.
Actually, our study shows that the average visible RCA vessel length of $114.9 \pm 29.3 \mathrm{~mm}$ compared favorably with earlier reported navigator-gated bSSFP $(80 \pm 40 \mathrm{~mm})$ and gradient echo sequences $(95 \pm 22 \mathrm{~mm})$ in RCA at 3.0 T (Kaul et al. 2004). Therefore, a large value of T2prep could improve image quality. Sahar et al. reported that T2 prep technic increased image quality compared to non T2 prep technic (Soleimanifard et al. 2013). Mueller et al. state that dual-source RF transmission with RF shimming results in an optimized SAR distribution, thereby reducing local SAR peaks (Mueller et al. 2012). As such, we can decrease SAR by using multitransmit technology.

Our study had a number of limitations. First, we only evaluated 10 healthy volunteers. Future studies are needed to evaluate more patients with suspected coronary heart disease. Second, our evaluation only involved a protocol for single-breath-hold whole-heart MRA with the recent 3-T systems. Future studies are needed to compare the protocol for a single breath hold and the protocol for free breathing with a respiratory gating method. Third, total scan time in our study is comparatively long (from $31 \mathrm{sec}$ to $45 \mathrm{sec})$. However, increasing reduction factor in parallel 
imaging be able to shorten total scan time in future studies. Forth, in our study, the image quality of the distal segments of the LCX and LAD were significantly poorer than the image quality of the RCA. When the distal segments were excluded, the image quality between the coronary arteries was not significant different. We believe that the poor quality of the LCX and LAD was caused by the small diameter of its distal segment. The result of the previous report was the same kind (Kim et al. 2006).

In conclusion, 3-T MRI with single-breath-hold 3D whole-heart coronary MRA can yield adequate image quality. Further study is needed to evaluate the clinical benefit of this technique.

\section{Competing interests}

The authors declare that they have no competing interests.

\section{Authors' contributions}

$\mathrm{YI}, \mathrm{TN}, \mathrm{MK}, \mathrm{TK}$ and TO made substantial contributions to conception and design, acquisition of data, analysis and interpretation of data; $\mathrm{YI}$ and TN involved in drafting the manuscript. Yl, TN, NS, KH and YY gave final approval of the version to be published. $\mathrm{YI}$ and TN agreed to be accountable for all aspects of the work in ensuring that questions related to the accuracy or integrity of any part of the work are appropriately investigated and resolved. All authors read and approved the final manuscript.

\section{Author details}

'Diagnostic Radiology, Amakusa Medical Center, Kameba 854-1, Amakusa, Kumamoto 863-0046, Japan. ${ }^{2}$ Department of Diagnostic Radiology, Graduate School of Medical Sciences, Kumamoto University, Honjo 1-1-1, Kumamoto, Kumamoto 860-8556, Japan. ${ }^{3}$ Department of Cardiovascular Internal Medicine, Amakusa Medical Center, Kameba 854-1, Amakusa, Kumamoto 863-0046, Japan. ${ }^{4}$ Department of Surgery, Amakusa Medical Center, Kameba 854-1, Amakusa, Kumamoto 863-0046, Japan. ${ }^{5}$ MR Clinical Scientist Philips Healthcare AsiaPacific, 13-37 Kohnan 2-chome Minato-ku, Tokyo 108-8507, Japan. ${ }^{6}$ Department of Diagnostic Radiology, Graduate School of Medical Sciences, Kumamoto University, Honjo 1-1-1, Kumamoto, Kumamoto 860-8556, Japan.

Received: 13 June 2014 Accepted: 5 November 2014

Published: 11 November 2014

\section{References}

Fischer SE, Wickline SA, Lorenz CH (1999) Novel real-time R-wave detection algorithm based on the vectorcardiogram for accurate gated magnetic resonance acquisitions. Magn Reson Med 42(2):361-370, doi:10.1002/(SICI)1522-2594

Kaul MG, Stork A, Bansmann PM, Nolte-Ernsting C, Lund GK, Weber C, Adam G (2004) Evaluation of balanced steady-state free precession (TrueFISP) and K-space segmented gradient echo sequences for 3D coronary MR angiography with navigator gating at 3 Tesla. Röfo 176(11):1560-1565, doi:10.1055/s-2004-813629

Kim YJ, Seo JS, Choi BW, Choe KO, Jang Y, Ko YG (2006) Feasibility and diagnostic accuracy of whole heart coronary MR angiography using free-breathing $3 \mathrm{D}$ balanced turbo-field-echo with SENSE and the half-fourier acquisition technique. Korean J Radiol 7(4):235-242

Lim RP, Winchester PA, Bruno MT, Xu J, Storey P, McGorty K, Sodickson DK, Srichai MB (2013) Highly accelerated single breath-hold noncontrast thoracic MRA: evaluation in a clinical population. Invest Radiol 48(3):145-151, doi:10.1097/RLI.0b013e31827994c2

Maintz D, Aepfelbacher FC, Kissinger KV, Botnar RM, Danias PG, Heindel W, Manning WJ, Stuber M (2004) Coronary MR angiography: comparison of quantitative and qualitative data from four techniques. AJR Am J Roentgenol 182(2):515-521, doi:10.2214/ajr.182.2.1820515

Makowski MR, Wiethoff AJ, Jansen CH, Uribe S, Parish V, Schuster A, Botnar RM, Bell A, Kiesewetter C, Razavi R, Schaeffter T, Greil GF (2012) Single breath-hold assessment of cardiac function using an accelerated 3D single breath-hold acquisition technique-comparison of an intravascular and extravascular contrast agent. J Cardiovasc Magn Reson 14:53, doi:10.1186/1532-429x-14-53

Mueller A, Kouwenhoven M, Naehle CP, Gieseke J, Strach K, Willinek WA, Schild $H H$, Thomas D (2012) Dual-source radiofrequency transmission with patientadaptive local radiofrequency shimming for 3.0-T cardiac MR imaging: initial experience. Radiology 263(1):77-85, doi:10.1148/radiol.11110347

Nassenstein K, Waltering KU, Kelle S, Schlosser T, Breuckmann F, Maderwald S, Hunold P, Nagel E, Barkhausen J (2008) Magnetic resonance coronary angiography with Vasovist: in-vivo T1 estimation to improve image quality of navigator and breath-hold techniques. Eur Radiol 18(1):103-109, doi:10.1007/s00330-007-0720-0

Nezafat R, Stuber M, Ouwerkerk R, Gharib AM, Desai MY, Pettigrew RI (2006) B1-insensitive T2 preparation for improved coronary magnetic resonance angiography at 3 T. Magn Reson Med 55(4):858-864, doi:10.1002/mrm.20835

Okada T, Kanao S, Kuhara S, Ninomiya A, Fujimoto K, Kido A, Togashi K (2011) Whole-heart coronary MR angiography under a single breath-hold: a comparative study with respiratory-gated acquisition using a multi-element phased-array coil. Clin Radiol 66(11):1060-1063, doi:10.1016/j.crad.2011.06.004

Ruipeng N, Yidong D, Guang Y, Gengying L (2009) A digital receiver with fast frequency- and gain-switching capabilities for MRI systems. MAGMA 22(6):333-342, doi:10.1007/s10334-009-0182-2

Sakuma H, Ichikawa Y, Suzawa N, Hirano T, Makino K, Koyama N, Van Cauteren M, Takeda K (2005) Assessment of coronary arteries with total study time of less than 30 minutes by using whole-heart coronary MR angiography. Radiology 237(1):316-321, doi:10.1148/radiol.2371040830

Scanlon PJ, Faxon DP, Audet AM, Carabello B, Dehmer GJ, Eagle KA, Legako RD, Leon DF, Murray JA, Nissen SE, Pepine CJ, Watson RM, Ritchie JL, Gibbons RJ, Cheitlin MD, Gardner TJ, Garson A Jr, Russell RO Jr, Ryan TJ, Smith SC Jr (1999) ACC/AHA guidelines for coronary angiography. A report of the American College of Cardiology/American Heart Association Task Force on practice guidelines (Committee on Coronary Angiography). Developed in collaboration with the Society for Cardiac Angiography and Interventions. J Am Coll Cardiol 33(6):1756-1824

Soleimanifard S, Schar M, Hays AG, Prince JL, Weiss RG, Stuber M (2013) Spatially selective implementation of the adiabatic T2Prep sequence for magnetic resonance angiography of the coronary arteries. Magn Reson Med 70(1):97-105, doi:10.1002/mrm.24437

Stuber M, Botnar RM, Fischer SE, Lamerichs R, Smink J, Harvey P, Manning WJ (2002) Preliminary report on in vivo coronary MRA at 3 Tesla in humans. Magn Reson Med 48(3):425-429, doi:10.1002/mrm. 10240

Wu YW, Tadamura E, Yamamuro M, Kanao S, Nakayama K, Togashi K (2007) Evaluation of three-dimensional navigator-gated whole heart MR coronary angiography: the importance of systolic imaging in subjects with high heart rates. Eur J Radiol 61(1):91-96

doi:10.1186/2193-1801-3-667

Cite this article as: lyama et al: Single-breath-hold whole-heart coronary MRA in healthy volunteers at 3.0-T MRI. SpringerPlus 2014 3:667.

\section{Submit your manuscript to a SpringerOpen ${ }^{\odot}$ journal and benefit from:}

- Convenient online submission

- Rigorous peer review

- Immediate publication on acceptance

- Open access: articles freely available online

- High visibility within the field

- Retaining the copyright to your article

Submit your next manuscript at $>$ springeropen.com 